\title{
A Novel Operating Strategy for Customer-Side Energy Storages in Presence of Dynamic Electricity Prices
}

\author{
Enrico Telaretti $^{1}$ - Eleonora Riva Sanseverino ${ }^{1} \cdot$ Mariano Ippolito $^{1}$. \\ Salvatore Favuzza ${ }^{1}$. Gaetano Zizzo ${ }^{1}$
}

Received: 3 March 2015 / Revised: 1 September 2015 / Accepted: 2 September 2015 / Published online: 14 September 2015

(C) Springer Science+Business Media Singapore 2015

\begin{abstract}
In the wholesale energy market, electricity prices are determined by the balance between supply and demand. Normally, customers are not exposed to these variations but pay a constant electricity price. In an attempt to reduce demand peaks, several utilities are moving from a conventional fixed-rate pricing scheme to a new market-based model, based on time-of-use or real-time pricing, able to closely reflect the wholesale energy price. Electricity customers can thus take profit from the installation of storage systems, shifting their energy consumption from on-peak to off-peak periods. This paper presents a novel charging strategy to manage customer storage systems in presence of hourly electricity prices. The optimal operating schedule of the storage device is obtained by maximizing an objective function which corresponds to the maximum benefit for the storage owner. The proposed method is developed under the assumption that the operating scheduling of the battery energy storage system (BESS) does not depend on the specific facility's consumption. The model can be applied to several kinds of storages although the simulations refer to a lead-acid battery. Test results show that the proposed
\end{abstract}

Enrico Telaretti

telaretti@dieet.unipa.it

Eleonora Riva Sanseverino

eleonora.rivasanseverino@unipa.it

Mariano Ippolito

ippolito@dieet.unipa.it

Salvatore Favuzza

favuzza@dieet.unipa.it

Gaetano Zizzo

zizzo@dieet.unipa.it

1 Department of Energy, Information Engineering and Mathematical Models, University of Palermo, Viale delle Scienze, 90128 Palermo, Italy operation strategy is effective to maximize the profit for the customer. The results also show that the investment can be recovered after several years when a lead-acid battery is used, but the situation will change in the near future, thanks to the cost reduction of storage technologies expected in coming years.

Keywords Battery energy storage system - Optimal operation - Load shifting - Real-time electricity price . Electricity market

\section{Introduction}

In the last years, the cost of generating electricity has risen around the world. For example, in the EU the average price of electricity for residential consumers has risen $6.92 \%$ from 1990 to 2010 [3]. The increase was particularly large in recent years, especially in those countries where the development of renewable plants has been very strong [12]. In this regard, Table 1 shows the increase in electricity prices in the last 3 years (2011-2013) for some EU countries, for residential and industrial sectors, respectively. It is impressive to observe that electricity prices have increased even more than $20 \%$ in the last 3 years, mainly due to the advantageous photovoltaic (PV) support policies adopted in such countries $[5,7,10,11]$.

In the wholesale energy market, electricity prices are determined by the balance between supply and demand. Normally, electricity users are not exposed to these fluctuations but pay a constant electricity price. In an attempt to reduce demand peaks, several utilities are moving from a conventional fixed-rate pricing scheme to new market-based models, where the electricity cost is left free to fluctuate depending on the balance between supply and demand. Such dynamic pricing schemes reflect the prices of the wholesale market 
Table 1 Increase in electricity prices in the last 3 years (2011-2013) in some EU countries

\begin{tabular}{|c|c|c|c|c|c|c|}
\hline & \multicolumn{6}{|c|}{ Electricity prices $(€ / \mathrm{kWh})$} \\
\hline & \multicolumn{2}{|l|}{ Greece } & \multicolumn{2}{|l|}{ Italy } & \multicolumn{2}{|l|}{ UK } \\
\hline & Household & Industry & Household & Industry & Household & Industry \\
\hline 2011 & 0.125 & 0.105 & 0.199 & 0.152 & 0.143 & 0.098 \\
\hline 2012 & 0.139 & 0.118 & 0.213 & 0.165 & 0.168 & 0.115 \\
\hline 2013 & 0.156 & 0.125 & 0.229 & 0.168 & 0.174 & 0.118 \\
\hline Increase 2011-2013 (\%) & 24.80 & 19.05 & 15.08 & 10.53 & 21.68 & 20.41 \\
\hline
\end{tabular}

and are able to lower demand peaks and the volatility of the wholesale prices [4]. A first example of dynamic pricing tariff is time-of-use (TOU) pricing, that provides two or three price levels for the customer (generally "on-peak", "mid-peak" and "off-peak" prices), depending on the time of day. Electricity users are advised in advance about electricity prices, that are not normally modified more than once or twice per year. A more flexible electricity pricing scheme is real-time pricing (RTP), for which the retail electricity price closely reflects the wholesale energy price. In this case, customer electricity prices may vary at any time of day, encouraging users to behave in an economically optimal way.

Figure 1 shows a comparison between the wholesale and the retail electricity prices, for Norway [20]. The difference between the two curves is the profit margin for the utility.

Normally, in the hourly spot market, the electricity price is known a day ahead, therefore it can be easily transferred by the utility to the final users. If this is the case, electricity customers could shift their energy consumption from high-price to low-price times of the day, thus reducing their electricity bills. However, in practice electricity users respond only partially to changes in the energy cost [22]. A more powerful way to reduce electricity bill is to install energy storage systems, able to shift energy consumption from on-peak to off-peak periods, thus avoiding to pay high electricity rates [13]. This can be accomplished by applying a load shifting strategy, charging the storage in low-price times and discharging it during high-price periods. In this context, California has already introduced a Resolution (Resolution E-4586 2012) that will implement a standardized Permanent Load Shifting (PLS) program in three major California's public utilities [25]. As defined by the California Resolution, PLS "refers to the shifting of energy usage from one period of time to another on a recurring basis, often by storing energy produced during off-peak hours and using the energy during peak hours to support loads". Examples of PLS technologies include ice thermal energy storages [6] and batteries. Significant advantages of PLS include the ability to defer electricity generation and transmission system upgrades, to reduce transmission congestion costs, to reduce peak demands and to lower the greenhouse gas emissions and the fuel consumption.

Storage units can also be used in conjunction with Renewable Energy Sources (RES), thereby reducing the impact of renewable technologies on the electricity network, or as an effective way to compensate for the variability of renewable generation [15]. Storage systems can also be operated so as to improve the value of RES power in the real-time market, in order to maximize the profit for the RES power producer [24]. In all these applications, custom devices need to be used
Fig. 1 Comparison between wholesale and retail prices

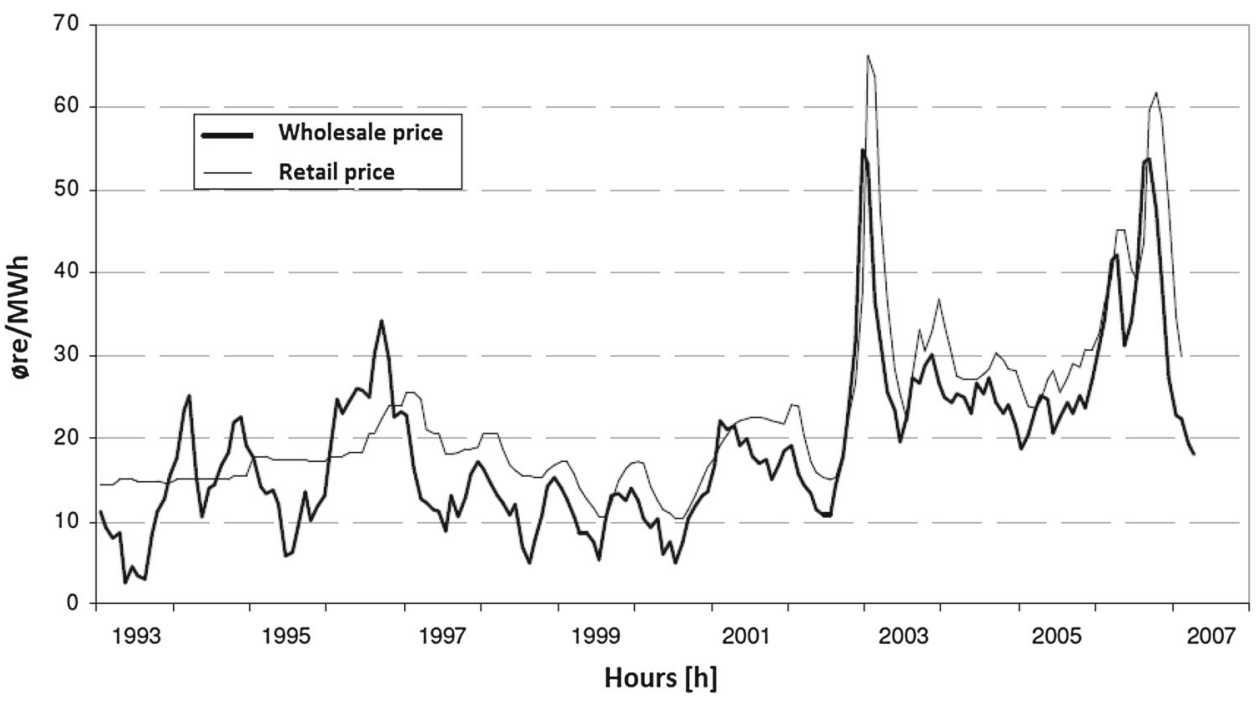


Fig. 2 Block diagram of electricity users interfaced with the DSO through a DRP

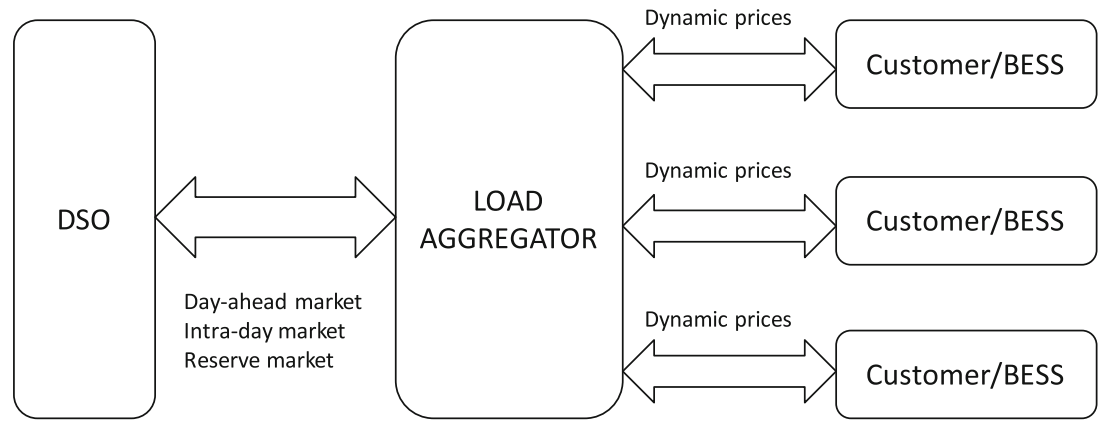

to ensure a proper interconnection and a reliable control system, according to the technical specifications of each country [18].

One of the main barriers for the participation of end-users to the electricity market is the minimum size limit established by network operators. In order to overcome this issue, small and widely dispersed customers can be grouped and managed by Demand Response Providers (DRP) working as load aggregators. DRP act as intermediaries between the Distribution System Operators (DSO) and the final users, submitting bids in the Day-Ahead, Intra-Day and in the Ancillary Services market. Doing so, the flexibility of all customers, even the smaller ones, can be exploited. Figure 2 shows the architecture of the system.

In this paper we present a novel charging strategy to manage a customer storage system in presence of dynamic tariffs. Differently from a previous work [29] where emphasis was placed in the test results, highlighting the changes in power profiles occurring as a result of the proposed battery energy storage system (BESS) charging strategy, in the present paper the main aim is to define, characterize and test the BESS operating strategy, by maximizing an objective function which expresses the customer benefit.

Traditionally, most of the studies address the optimal operation of a storage system based on dynamic programming $[17,23,30]$, linear programming [33], nonlinear programming $[16,26]$, multipass iteration particle swarm optimization approach [21] and Kalman filter [32].

Unlike the studies listed above, often requiring an estimate of the end-user profile, the proposed operation strategy is able to properly identify, for each daily period, the charging and discharging times, relying only on the hourly spot market price profile, regardless on the specific facility's load profile. This is made possible since the storage is sized referring to the facilities' energy consumption during peak hours, on the day of the year of lower consumption. Under this assumption, the storage will be able to supply the entire peak load during the day of the year of lower consumption, but only a portion of the peak load on the other days. The proposed operating strategy is simple and effective and has a low computational burden. The proposed approach can be applied to several kinds of storage systems but a lead-acid battery has been chosen for the simulations.

The remainder of the paper is organized as follows. In "Italian Electricity Market" section, the Italian Electricity Market is outlined, with specific reference to the Day-Ahead Market. In "Energy System Description and Operational Assumptions" section, the customer energy system used in this paper is briefly described and the basic operational assumptions are outlined. In "Problem Formulation" section, the problem formulation is provided, showing the objective function to be maximized. In "The Proposed Operating Strategy" section, the proposed storage operating strategy is developed. In "Cost Benefit Analysis" section, the costbenefit analysis is presented, by defining the storage costs and the savings for the customer. In "Case Study" section, a case study is presented and the performance of the algorithm is evaluated for the base case. Finally, in last section, the conclusion of the work is summarized.

\section{Italian Electricity Market}

In Italy, the liberalization of the electricity market started in the 1990s with the enactment of Legislative Decree n. 79 of 16 March 1999 [19], with the purpose of creating a single electricity market, promoting competition in electricity generation, sale and purchase, under criteria of neutrality, transparency and objectivity.

The Electricity Market consists of [14];

- a spot market (Mercato Elettrico a Pronti, MPE);

- a forward market (Mercato Elettrico a Termine, MTE);

- a platform for physical delivery of financial contracts concluded on IDEX (CDE).

In the MTE, participants can negotiate forward electricity contracts with delivery and withdrawal obligation, with GME (Italian Electricity Market Operator) acting as a central counterparty. Two types of contracts can be negotiated, base-load or peak-load, with monthly, quarterly and yearly delivery periods. 
MPE consists of three submarkets:

- the Day-Ahead Market (Mercato del Giorno Prima, MGP);

- the Intra-Day Market (Mercato Infragiornaliero, MI);

- the Ancillary Services Market (Mercato per il Servizio di Dispacciamento, MSD).

MGP is an auction market where hourly energy blocks can be negotiated for the next day. Most of the electricity sale and purchase transactions take place in this market.

Entitled participants negotiate in the MGP by submitting demand bids (supply offers), each one composed by a pair of values, i.e. quantity and maximum (minimum) price at which they are willing to purchase (sell).

Offers/bids are accepted according to the economic meritorder criterion, respecting the transmission capacity limits between zones. In particular:

- all accepted supply offers are valued at the clearing price of the zone to which they belong to. The clearing price is determined, for each hour, by the intersection between the supply and the demand curve and it is different for each market zone;

- the accepted demand bids are valued at the single national price (Prezzo Unico Nazionale, PUN), defined as the average of zonal prices weighted by zonal consumptions. Therefore, utility customers purchase electricity at the PUN value.

PUN values are quite volatile, due to the random changes in generation and demand in the Day-Ahead market, as clearly shown in Fig. 3, where the PUN values in the year 2014 are drawn. Figure 3 clearly shows the strong and unpredictable variability of the single national price [8].

Since, in the hourly spot market, electricity prices are known a day ahead, participants have the option to modify their MGP programs by submitting additional offers in the MI. In this case, all market transactions are valued at the zonal marginal price. The volume of transactions traded on MI is significantly lower than that on MGP.

Both MGP and MI are marginal price markets, namely the electricity price reflects the value of energy in the specific location at the time that it is delivered. Differently from MGP and MI, MSD is a pay-as-bid market, where the Transmission System Operator (TSO) procures the necessary resources for operating and monitoring the power system [9]. MSD consists of two submarkets: a scheduling phase ("MSD ex-ante") and a balancing market (MB) both aimed at relief the intrazonal congestions, create the energy reserves and ensure the real-time balance between generation and demand.

\section{Energy System Description and Operational Assu- mptions}

The customer energy system consists of a passive user and a BESS, interconnected to the power grid through a bidirectional converter, as depicted in Fig. 4. The bidirectional converter consists of a rectifier AC/DC (the battery charger) and an inverter DC/AC. The electronic switches, $\mathrm{S} 1$ and S2, interface the battery bank with the conversion device by means of a control system, managing the charging/discharging time of the battery.

The battery system is handled in order to ensure an economic benefit for the customer, through a load shifting strategy. Since the PUN value is available one day ahead and is defined each hour, the elementary time interval used in the

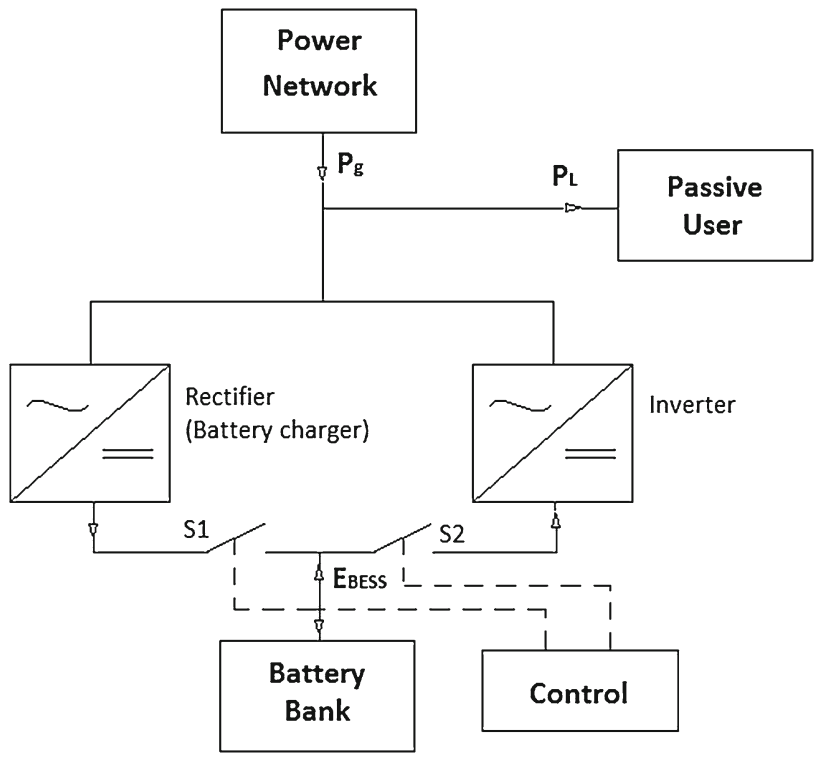

Fig. 4 Grid-connected customer energy system operating in parallel with the storage system
Fig. 3 The hourly PUN value in the year 2014

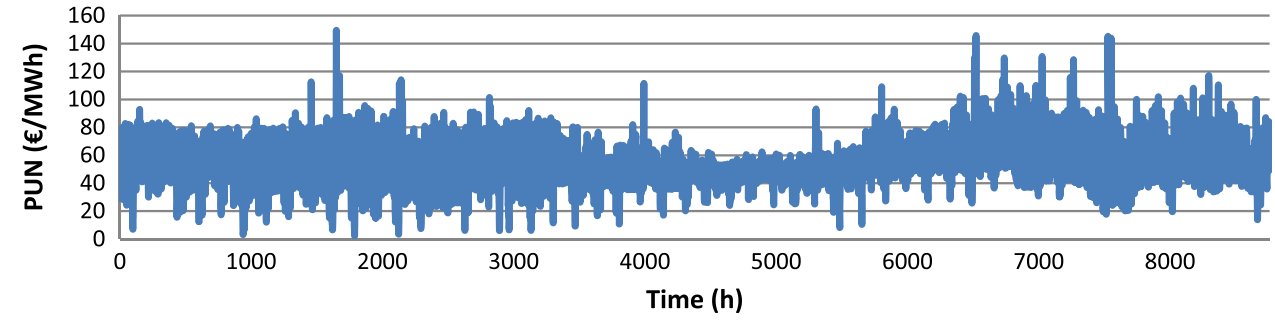


study is $1 \mathrm{~h}$. In addition, the reference period is one day, i.e. the battery operation is defined starting from a vector of 24 elements as input data.

Three different operating modes are considered for the storage system:

- charging mode, activated when the electricity prices are low;

- standby mode, in which the power grid supplies directly the end-user without contribution of the storage;

- discharging mode, activated when the electricity prices are high, where part of the load is supplied from the battery.

The following assumptions have been made:

- the end-user is allowed to buy the consumed energy at a hourly tariff (RTP tariff), defined by the utility on a daily basis. The RTP scheme is assumed to be proportional to the PUN values, by applying a percentage increase, in order to ensure a benefit for the utility and account for taxes;

- the use of the storage device does not influence the price of electricity in the energy market (small price taking storage devices);

- the common frictions of the battery operation are accounted for by incorporating imperfect charging efficiency. The discharging efficiency is neglected;

- the charge/discharge time is assumed to be constant and equal to the time step (i.e. $1 \mathrm{~h}$ ), and the battery is always discharged at the same depth-of-discharge (DOD);

- at the end of each charge/discharge cycle the battery returns to the initial state-of-charge (SOC). Doing so the battery energy constraint is automatically satisfied, i.e. the storage level cannot exceed the rated energy capacity of the device, at any time;

- the charge/discharge rate of the battery is assumed constant in each time interval. Doing so, the storage charging/discharging constraint is automatically satisfied, i.e. the energy charged/discharged cannot be more than the rated power capacity of the device, at any time;

- the optimum storage capacity is assumed equal to the facilities' energy consumption during peak hours [34], on the day of the year of lower consumption. In other words, the battery is sized so that it can supply the entire peak load during the day of the year of lower consumption, and only a portion of the peak load on the other days. Doing so, the BESS can be operated regardless on the specific facility's load profile and, at the same time, the minimum installation cost is assured. As a consequence of this statement, the power flow is always directed from the grid to the load and the stored energy can only be used for load compensation, without selling to the utility;
In addition to the above mentioned hypotheses, the battery self-discharge is disregarded and the battery capacity is assumed constant throughout the battery life, without degradation.

\section{Problem Formulation}

Since, in the hourly spot market, the electricity price is know a day ahead, the storage owner may schedule the battery operation in order to take profit from the gap between high and low electricity prices.

The objective of the load shifting strategy is to find a proper battery charge/discharge/standby schedule, for each daily period, in order to minimize the total cost for the enduser. The last can be expressed as follows:

$C_{i}=\sum_{t=1}^{24} R T P_{i}(t) \cdot E_{g}^{i}(t)$

where:

- $C_{i}$ is the total cost for the end-user at the day $i$;

- $R T P_{i}(t)$ is the customer RTP tariff at the hour $t$ of the day $i$;

- $E_{g}^{i}(t)$ is the energy absorbed from the grid at the hour $t$ of the day $i$.

The expression (1) can be written as:

$C_{t}=\sum_{t=1}^{24} R T P_{i}(t) \cdot E_{L}^{i}(t)-\sum_{t=1}^{24} R T P_{i}(t) \cdot E_{B E S S}$

where:

- $E_{L}^{i}(t)$ is the customer energy consumption at the hour $t$ of the day $i$;

- $E_{B E S S}$ is the energy charged/discharged from the storage system (neglecting friction during the battery operation).

The energy discharged, $E_{B E S S}$, is assumed positive if directed towards the load, negative otherwise.

Since the operating strategy does not depend on the facility's load profile and the battery is assumed to be a small price taking storage device, the objective function can then be written as:

$\max \sum_{t=1}^{24} R T P_{i}(t) \cdot E_{B E S S}$

Since the charging/discharging power, $P_{B E S S}$ and the DOD are constant in our evaluations, the maximization of the 
Fig. 5 Typical RTP daily profile and its average value

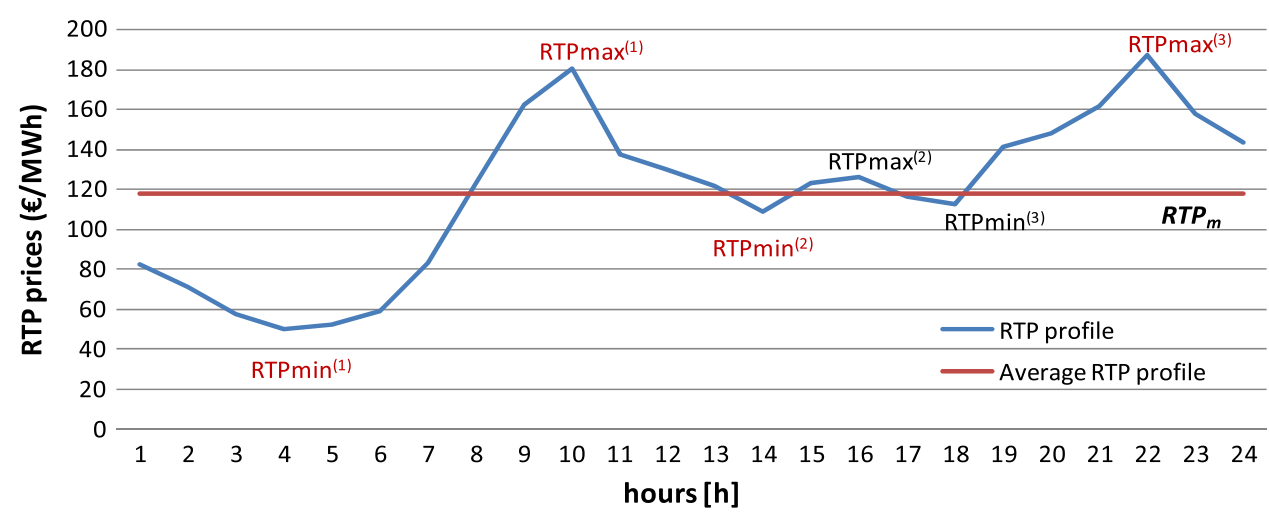

objective function corresponds to determine the optimal charging/discharging scheduling, in relation to the RTP values changing every day.

\section{The Proposed Operating Strategy}

The operating strategy is able to uniquely determine the daily charging/discharging intervals of the storage device so as to maximize the economic saving for the customer, according to (3). The algorithm then handles a vector of 24 elements as input data, that represents the daily RTP profile, as drawn in Fig. 5 [14]. The same figure also shows the daily average RTP value $\left(R T P_{m}\right)$.

The discharging revenue and the charging cost can be represented as follows:

$E_{B E S S} \cdot R T P_{\max }$

$E_{B E S S} / \mu \cdot R T P_{\text {min }}$

where $R T P_{\text {min }}$ and $R T P_{\text {max }}$ are the RTP prices associated with minimum and maximum RTP values and $\mu$ is the charging efficiency.

Because the BESS operation is profitable only when the discharging revenue is greater than the charging cost, we have:

$E_{B E S S} \cdot R T P_{\max }>E_{B E S S} / \mu \cdot R T P_{\min }$

Ultimately, the battery operation can make some profits only when the following inequality condition is satisfied:

$\mu \cdot R T P_{\max }>R T P_{\min }$

If condition (7) is not satisfied, the BESS charging losses are greater than the saving arising from the load shifting strategy, then the battery will remain in standby state [16].

The generic hourly interval can assume three different operating states:
- standby state;

- potential charging/discharging state;

- active charging/discharging state.

The algorithm starts by assigning all the 24 intervals an initial state of standby.

The following assumptions are also needed:

- each interval can first switch from the initial standby state to a potential charging/discharging state; from this latter state, the interval can switch to an active charging/discharging state or return to a standby state depending on the fulfillment of condition (7). When an interval is assigned an active charging/discharging state, this one becomes definitive;

- at the end of the algorithm processing, only two operating states can be associated to each elementary time interval: standby or active charging/discharging.

Figure 6 shows the flow chart of the proposed operating strategy. The battery is considered discharged at the start of the process, but the procedure is dual if the BESS is assumed charged. The procedure can be summarized as below.

(1) Firstly, the algorithm processes the 24 element vector by determining the average RTP value $\left(R T P_{m}\right)$. By comparing the daily RTP profile with the average RTP value, the min/max RTP values are derived (see Fig. 5):

$R T P_{\min }^{(k)}, \quad R T P_{\max }^{(j)} \quad \forall k, j: 1 \leq k \leq N ; 1 \leq j \leq M$

being $N, M$ the total number of $\min / \max$ RTP values per day, and $k, \mathrm{j}$ indexes denoting the number of minimum/maximum RTP values per day, respectively. The algorithm starts by considering the first pair of consecutive $\mathrm{min} / \max$ RTP values, switching their status to potential.

(2) If the RTP values satisfy condition (7) (as in the case of $R T P_{\min }^{(1)}, R T P_{\max }^{(1)}$, in Fig. 5), they are definitively switched in active state and the algorithm moves to the next pair 
Fig. 6 Flow chart of the proposed operating strategy

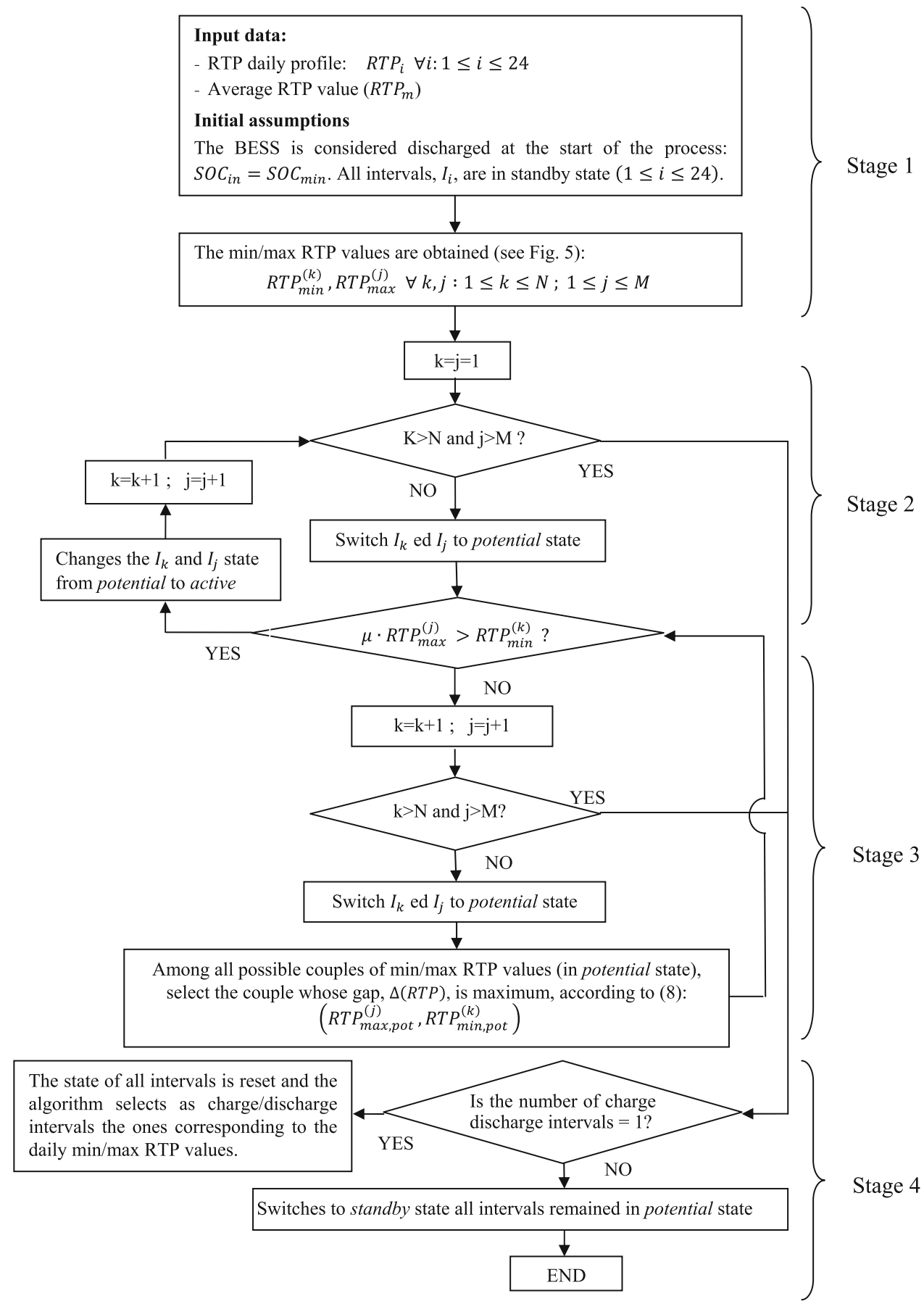

of $\min / \max$ RTP values. Conversely, if condition (7) is not satisfied (as in the case of $R T P_{\min }^{(2)}, R T P_{\max }^{(2)}$ ), the couple of RTP values retains the state of potential, and the algorithm moves to the next pair of consecutive $\mathrm{min} / \mathrm{max}$ RTP values $\left(R T P_{\min }^{(3)}, R T P_{\max }^{(3)}\right)$, switching their status to potential.

(3) The algorithm then checks all the possible couples of $\mathrm{min} / \mathrm{max}$ RTP values remained in potential state $\left(R T P_{\min }^{(2)}\right.$, $\left.R T P_{\max }^{(2)}, R T P_{\min }^{(3)}, R T P_{\max }^{(3)}\right)$ identifying the couple whose gap, $\Delta(R T P)$, is maximum $\left(R T P_{\min }^{(2)}\right.$ and $R T P_{\max }^{(3)}$ in Fig. 5):

$$
\Delta(R T P)=R T P_{\max , p o t}^{(j)}-R T P_{\min , p o t}^{(k)} \text { is maximum }
$$

where $R T P_{\text {min, pot }}^{(k)}, R T P_{\text {max, pot }}^{(j)}$ is the generic couple of $\min / \max$ RTP values remained in potential state, with $k \leq j$ (the minimum comes before the maximum). 
If the couple of min/max RTP values that satisfies (8) fulfills condition (7) (as in the case of $R T P_{\min }^{(2)}, R T P_{\max }^{(3)}$ ), the algorithm definitively switches the corresponding timeslots to active state, moving to the next pair of consecutive $\mathrm{min} / \mathrm{max}$ RTP values (restarting from step 2). If (7) is not satisfied, the corresponding timeslots retain the state of potential and the algorithm moves to the next pair of min/max RTP values, switching their status to potential (restarting from step 3 ). The procedure ends when the last couple of $\min / \max$ RTP values is counted ( $i=24$ i.e. $k=N$ and $j=M$ ).

Finally, the algorithm checks if the number of charging/discharging intervals per day is equal to one. If this is the case, the state of all intervals is reset and the algorithm identifies as charging interval the one corresponding to the daily minimum RTP value $\left(R T P_{\text {min }}^{(1)}\right.$ in Fig. 5) and as discharging interval the one corresponding to the daily maximum RTP value (RTP max $^{(3)}$ in Fig. 5).

Following the RTP profile in Fig. 5, the strategy steps are processed in the following sequence:

Step 1 The algorithm starts the procedure by checking if the first couple of RTP values $\left(R T P_{\min }^{(1)}, R T P_{\max }^{(1)}\right)$ satisfies (7). Since (7) is satisfied, $R T P_{\text {min }}^{(1)}$ and $R T P_{\text {max }}^{(1)}$ are definitively switched to active state.

Step 2 The algorithm checks (7) for the second couple of RTP values $\left(R T P_{\min }^{(2)}, R T P_{\max }^{(2)}\right)$. Since (7) is not satisfied, $R T P_{\min }^{(2)}$ and $R T P_{\max }^{(2)}$ are maintained in potential state and the algorithm switches the third couple, $\left(R T P_{\text {min }}^{(3)}, R T P_{\max }^{(3)}\right)$ in potential state. At this time, both the second and the third couple of RTP values are in potential state;

Step 3 Among all possible combinations of min/max RTP values (in potential state), the algorithm identifies the couple whose gap is maximum (for which (8) is satisfied): $R T P_{\text {min }}^{(2)}$ and $R T P_{\text {max }}^{(3)}$. Since this couple satisfies (7), the corresponding timeslots are definitively switched to active state. The procedure ends as there are no other RTP values following. At this time, all intervals remained in potential state $\left(R T P_{\max }^{(2)}, R T P_{\text {min }}^{(3)}\right)$ are switched to standby state.

At the end of the algorithm processing, the active charging/discharging intervals are the ones marked in red in Fig. 5. It is worth noting that, if $R T P_{\text {min }}^{(k)}, R T P_{\text {max }}^{(j)}$ had been calculated only according to consecutive $\mathrm{min} / \mathrm{max}$ RTP values, the active states would be the couples $\left(R T P_{\min }^{(1)}, R T P_{\max }^{(1)}\right)$ and $\left(R T P_{\min }^{(3)}, R T P_{\max }^{(3)}\right)$, which do not correspond to any optimal condition.

The flow chart in Fig. 6 has been divided into four stages:

Stage 1 This is a preliminary stage, in which the input data are provided. In this stage the average value, $R T P_{m}$, and the $\min / \max$ RTP values, $\left(R T P_{\min }^{(k)}, R T P_{\max }^{(j)}\right)$ are derived.
Stage 2 In this stage, only one pair of $\mathrm{min} / \mathrm{max}$ RTP values at a time is switched to potential state, by checking if condition (7) is satisfied.

Stage 3 This stage is activated only if condition (7) is not satisfied. If this is the case, two couples of min/max RTP values are simultaneously switched to potential state, and condition (8) is checked.

Stage 4 The algorithm checks if the number of charging/discharging intervals per day is equal to one. If this is the case, the state of all intervals is reset and the algorithm selects as charge/discharge intervals the ones corresponding to the daily $\mathrm{min} / \mathrm{max}$ RTP values.

\section{Cost Benefit Analysis}

The economic analysis is performed by calculating the payback period (PBP) of the investment. PBP estimates the time required to recover the principal amount of an investment. It can be expressed as the minimum value of the index $n$ that satisfies the following inequality condition:

$\sum_{n=1}^{P B P} \frac{R_{a n}}{(1+i)^{n}} \geq C_{T O T}$

where:

- $R_{a n}$ is the annual customer saving arising from the load shifting strategy of the BESS, in year $n$;

- $C_{T O T}$ is the BESS investment cost;

$-i$ is the discount factor, assumed equal to $3 \%$.

The daily saving arising from the load shifting strategy of the BESS can be expressed as:

$$
\sum_{p} E_{B E S S} \cdot\left(R T P_{\max , p}^{(j)}-\frac{R T P_{\min , p}^{(k)}}{\mu}\right)
$$

being $R T P_{\text {max }, p}^{(j)}$ and $R T P_{\text {min, } p}^{(k)}$ the active charge/discharge intervals of the BESS (marked in red in Fig. 5) and $p$ an index denoting the number of operating cycles per day. The annual customer saving, $R_{a n}$, can finally be calculated by summing all the daily savings.

In order to calculate the PBP of the investment, the BESS capital cost, $C_{T O T}$, must be estimated. The total capital cost of the BESS can be expressed as the sum of the power conversion system (PCS) cost $\left(C_{P C S}\right)$, the storage cost $\left(C_{S T O R}\right)$ and the balance-of plants $(\mathrm{BOP})$ cost $\left(C_{B O P}\right)$ [27]:

$C_{T O T}=C_{P C S}+C_{S T O R}+C_{B O P}$ 
BOP includes the auxiliary components outside of the storage system. These costs include building construction, battery and PCS installation, interconnections, heating, ventilating, etc.

For most of the BESS, storage cost is proportional to the energy capacity, whereas PCS and BOP costs are proportional to the power rating. To account for this, equation (11) can be modified as follows:

$$
\begin{aligned}
C_{T O T}= & C_{P C S}^{u} \cdot P(\mathrm{~kW})+C_{S T O R}^{u} \cdot E(\mathrm{kWh}) \\
& +C_{B O P}^{u} \cdot P(\mathrm{~kW})
\end{aligned}
$$

where:

- $C_{P C S}^{u}$ and $C_{B O P}^{u}$ are the per unit costs of the PCS and of the BOP $(€ / \mathrm{kW})$, respectively;

- $C_{S T O R}^{u}$ is the per unit cost of the storage unit ( $\left.€ / \mathrm{kWh}\right)$.

- $E(\mathrm{kWh})$ and $P(\mathrm{~kW})$ are the energy capacity and the power rating of the BESS, respectively.

The specific cost of a battery depends on the chosen technology. Table 2 shows the main BESS parameters and the components cost for the selected lead-acid battery, according to [2]. The efficiency of the battery is assumed equal to $85 \%$ [1] and the number of cycles to failure (at $80 \%$ DOD) is 1250 [2].

Fixed and variable operation and management (O\&M) costs of the BESS have not been taken into account in the economical evaluation.

\section{Case Study}

The analysis has been carried out by referring to a typical medium-scale public facility (DEIM-Department of Energy, Information Engineering and Mathematical Mod-

Table 2 Main parameters and per unit costs of the selected lead-acid battery

\begin{tabular}{ll}
\hline Components & Specifications \\
\hline Technology & Lead-acid battery \\
Energy capacity $(\mathrm{kWh})$ & 10 \\
DOD per cycle $(\%)$ & 80 \\
Charging/discharging efficiency $(\%)$ & 85 \\
Number of cycles/year & 626 \\
Cycles to failure $(80 \%$ DOD) & 1250 \\
Plant Life (years) & 2 \\
$C_{B E S S}^{u}(€ / \mathrm{kWh})$ & 171 \\
$C_{P C S}^{u}(€ / \mathrm{kW})$ & 172 \\
$C_{B O P}^{u}(€ / \mathrm{kW})$ & 70 \\
\hline
\end{tabular}

els, University of Palermo). The operating strategy has been implemented by assuming the customer RTP tariff equals to the PUN values applied in Italy in 2014 , increased by a factor of 3, to incorporate the benefit for the utility and taxes. For the selected facility, eight different seasonal daily tariff profiles have been considered (PUN values for weekdays and weekends and for each season). The profiles were obtained by averaging the PUN values hour by hour, for each seasonal period. The considered PUN profiles are shown in Fig. 7a, b, for weekdays and weekends respectively.

One can observe that weekdays PUN values have a first price peak at about 09:00-10:00 a.m. and a second peak at 6:00-9:00 p.m. Conversely, weekends PUN values retain only the second peak at about 7:00-9:00 p.m. The first peek is, indeed, very flattened. As a result of this, the BESS will be charged two times on weekdays, only one time on weekends.

The optimal charging/discharging intervals of the BESS for each of the seasonal daily RTP profiles are listed in Table 3.

Finally, Table 4 illustrates the annual revenue, the capital cost and the PBP of the BESS.

It can be seen that the PBP is significantly higher than the battery life. The results could be even less favorable if fixed and variable O\&M costs would be considered. Therefore, lead-acid batteries cannot be profitably used in load shifting applications at the current prices of storage technologies. The situation could change in the near future, thanks to the cost reduction of storage systems expected in the coming years. Indeed, in [31] the authors provide the ranges of capital costs for the year 2011 and the expected costs for 2020, obtained from an extensive literature review and through many consultations with domain experts. The authors conclude that, especially for less mature technologies, there is significant room for cost and performance improvements. For example, for the Li-ion technology, a medium cost reduction of almost $50 \%$ is expected. A quicker reduction of storage costs could also be fostered by the introduction of support programs for the development of storage units, as was done in Germany in 2013.

An affordable operation of the BESS could also be obtained by increasing the gap between maximum and minimum RTP prices. In addition, the participation of BESS to the intra-day market could also increase the profitability of BESS in arbitrage services, as demonstrated in [28]. Then, a future paper will focus on an optimal operating strategy of the BESS in the intra-day electricity market.

\section{Conclusion}

This paper has presented a novel charging strategy to manage customer storage systems in presence of hourly electricity prices. The analysis has been carried out by referring to a 
Fig. 7 Seasonal PUN profiles for weekdays (a) and weekends (b)

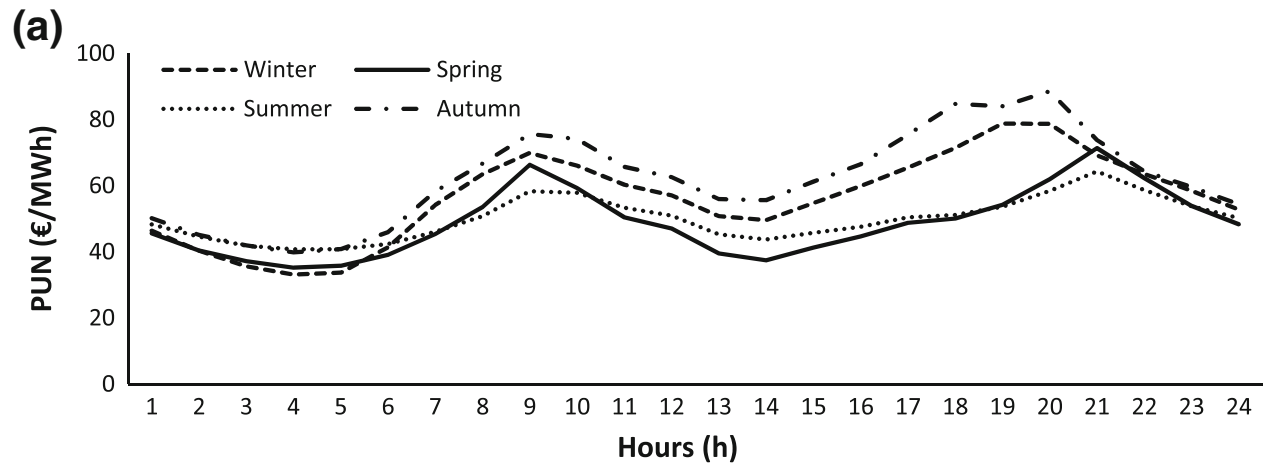

(b)

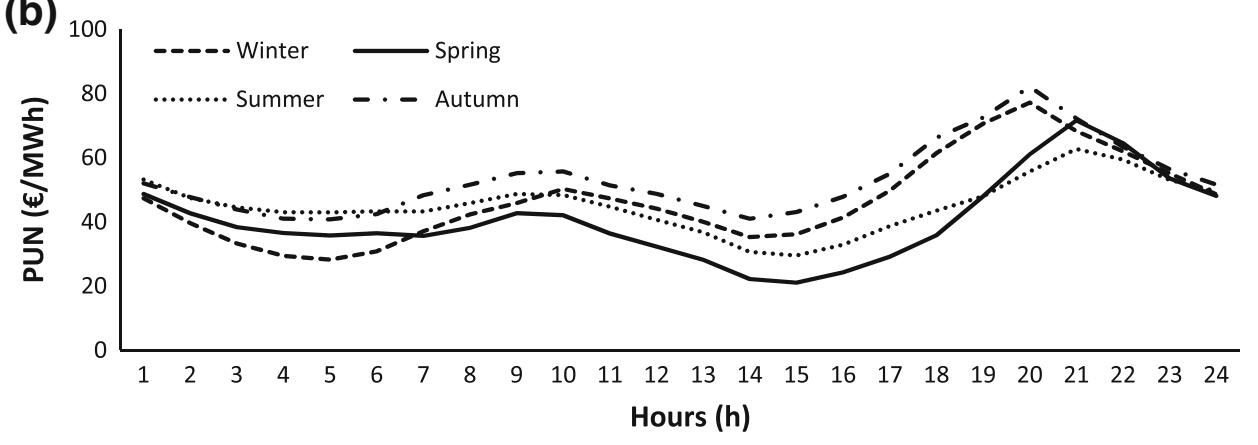

Table 3 Optimal charging/discharging intervals of the BESS for each seasonal daily RTP profiles

\begin{tabular}{lllll}
\hline & Charging hours & Discharging hours & Charging hours & Discharging hours \\
\hline Winter-weekdays & $03: 00-04: 00$ & $08: 00-09: 00$ & $13: 00-14: 00$ & $13: 00-20: 00$ \\
Spring-weekdays & $03: 00-04: 00$ & $08: 00-09: 00$ & $13: 00-14: 00$ & $20: 00-21: 00$ \\
Summer-weekdays & $03: 00-04: 00$ & $9: 00-10: 00$ & $13: 00-14: 00$ & $20: 00-21: 00$ \\
Autumn-weekdays & $03: 00-04: 00$ & $8: 00-09: 00$ & & $19: 00-20: 00$ \\
Winter-weekends & $04: 00-05: 00$ & $19: 00-20: 00$ & & \\
Spring-weekends & $14: 00-15: 00$ & $20: 00-21: 00$ & & \\
Summer-weekends & $14: 00-15: 00$ & $20: 00-21: 00$ & & \\
Autumn-weekends & $04: 00-05: 00$ & $19: 00-20: 00$ & \\
\hline
\end{tabular}

Table 4 Annual revenue, capital cost and PBP of the BESS

\begin{tabular}{lr}
\hline Annual revenue $(€)$ & 390 \\
Capital cost $(€)$ & 4130 \\
PBP & 13 \\
\hline
\end{tabular}

typical medium-scale public facility. The proposed management strategy aims to maximize the profit of the storage owner (electricity customer) by determining the optimal charge/discharge schedule. The optimal operating schedule of the storage device is obtained by maximizing an objective function which corresponds to the maximum benefit for the storage owner. Unlike the studies reported in the literature, often requiring an estimate of the end-user load profile, the proposed operating strategy is able to identify the proper charging schedule of the device regardless of the specific facility's load profile. This is made possible since the storage is sized referring to the facilities' energy consumption dur- ing peak hours, on the day of the year of lower consumption. Under this assumption, the storage will be able to supply the entire peak load during the day of the year of lower consumption, but only a portion of the peak load on the other days. Therefore, the optimal operating schedule of the storage device is obtained using a heuristic approach, without the need to estimate the customer's daily power profile. This could be particularly useful when the customer load profile cannot be scheduled with sufficient reliability, because of the uncertainty inherent in load forecasting. Notwithstanding, the same problem could be tackled with a more rigorous approach taking into account the estimated facilities' power profile, e.g. exploiting an optimization game theory between two competing entities, the customer power consumption against the storage device-based consumption. The model can be applied to several kind of storage systems but a leadacid battery has been chosen for the economical evaluations. 
Simulation results show that the investment can be recovered after several years (higher than the storage lifetime) when a lead-acid battery is used in the Italian context. Therefore, lead-acid technology cannot be profitably used in load shifting applications at the current hourly spot prices applied in Italy. The situation could change in the near future thanks to the cost reduction of storage technology expected in the coming years. A cost-effective operation of the BESS could be obtained by increasing the gap between maximum and minimum RTP prices or introducing support programs for the development of storage units.

The model is suitable to be used also considering the CDE, that is the platform where financial electricity derivatives contracts, concluded on IDEX, are executed.

In this case, the customer could also have influence on the electricity price, facilitating situations in which the algorithm will drive to an optimal solution.

The profitability of BESS in arbitrage services could also be increased through participation of BESS in the intra-day market. In a future paper, the authors will develop an optimal operating strategy of the BESS in the intra-day electricity market, will extend the economic evaluation to other storage technologies and will consider also the presence of a very large number of distributed storage systems. Indeed, in this last case, the proposed method will have to be modified, in order to avoid the simultaneous charge of all the storage systems that could give place to very high power peaks and instability situations.

Acknowledgments This work was supported by the project i-NEXT (Innovation for greeN Energy and eXchange in Transportation), identification code: PON04a2_Hi-NEXT (CUP B71H12000700005).

\section{References}

1. Akhil, A.A., Huff, G., Currier, A.B., Kaun, B.C., Rastler, D.M., Chen, S.B., Cotter, A.L., Bradshaw, D.T.: Gauntlett WD (2013) Electricity Storage Handbook in Collaboration with NRECA, Technical Report. SAND2013-5131. Albuquerque (2013)

2. Battke, B., Schmidt, T.S., Grosspietsch, D., Hoffmann, V.H.: A review and probabilistic model of life cycle costs of stationary batteries in multiple applications. Renew Sustain Energy Rev 25, 240-250 (2013)

3. Bertoldi, P., Hirl, B., Labanca, N.: Energy Efficiency Status Report 2012. JRC Scientific and Policy Report. Report EUR 25405 EN, European Union (2012)

4. Borenstein, S.: The long-run efficiency of real-time electricity pricing. Energy J 26(3), 93-116 (2005). doi:10.5547/ ISSN0195-6574-EJ-Vol26-No3-5

5. Braun, M., Stetz, T., Bründlinger, R., Mayr, C., Ogimoto, K., Hatta, H., Kobayashi, H., Kroposki, B., Mather, B., Coddington, M., Lynn, K., Graditi, G., Woyte, A., MacGill, I.: Is the DistributionGrid ready to accept large scale photovoltaic deployment?-state of the art, progress and future prospects. Prog Photovolt Res Appl 20(6), 681-697 (2011). doi:10.1002/pip.1204
6. Campoccia, A., Dusonchet, L., Telaretti, E., Zizzo, G.: Economic impact of ice thermal energy storage systems in residential buildings in presence of double-tariffs contracts for electricity. In: Proceedings of IEEE International Conference on the European Energy Market (EEM'09), Leuven, Belgium, 27-29 May 2009, pp. 1-5. doi:10.1109/EEM.2009.5207123 (2009)

7. Campoccia, A., Dusonchet, L., Telaretti, E., Zizzo, G.: An analysis of feed' in tariffs for solar PV in six representative countries of the European Union. Sol Energy 107(3), 530-542 (2014). doi:10. 1016/j.solener.2014.05.047

8. Clò, S., Cataldi, A., Zoppoli, P.: The merit-order effect in the Italian power market: the impact of solar and wind generation on national wholesale electricity prices. Energy Policy 77, 79-88 (2015). doi:10.1016/j.enpol.2014.11.038

9. Dotoli, M., Epicoco, N., Falagario, M., Sciancalepore, F., Costantino, N.: A Nash equilibrium simulation model for the competitiveness evaluation of the auction based day ahead electricity market. Comput Ind 65, 774-785 (2014). doi:10.1016/j.compind. 2014.02.014

10. Dusonchet, L., Telaretti, E.: Economic analysis of different supporting policies for the production of electrical energy by solar photovoltaics in eastern European Union countries. Energy Policy 38, 4011-4020 (2010). doi:10.1016/j.enpol.2010.03.025

11. Dusonchet, L., Telaretti, E.: Economic analysis of different supporting policies for the production of electrical energy by solar photovoltaics in western European Union countries. Energy Policy 38, 3297-3308 (2010). doi:10.1016/j.enpol.2010.01.053

12. Dusonchet, L., Telaretti, E.: Comparative economic analysis of support policies for solar PV in the most representative EU countries. Renew Sustain Energy Rev 42, 986-998 (2015). doi:10.1016/j.rser. 2014.10.054

13. Dusonchet, L., Ippolito, M.G., Telaretti, E., Graditi, G.: Economic impact of medium-scale battery storage systems in presence of flexible electricity tariffs for end-user applications. In: Proceedings of IEEE International Conference on the European Energy Market (EEM'12), Florence, Italy, 10-12 May 2012, pp. 1-5. doi:10.1109/ EEM.2012.6254685 (2012)

14. GME home page: http://www.mercatoelettrico.org/it/Default. aspx. Accessed 15 Sept 2014

15. Gu, Y., McCalley, J., Ni, M., Bo, R.: Economic modeling of compressed air energy storage. Energies 6, 2221-2241 (2013). doi:10. 3390/en6042221

16. Hu, W., Chen, Z., Bak-Jensen, B.: Optimal operation strategy of battery energy storage system to real-time electricity price in Denmark. In: Proceedings of the IEEE Power and Energy Society General Meeting, 25-29 July 2010, Minneapolis, pp. 1-7. doi:10. 1109/PES.2010.5590194 (2010)

17. Huang, T., Liu, D.: Residential energy system control and management using adaptive dynamic programming. In: Proceedings of the International Joint Conference on Neural Networks (IJCNN'11), San Jose, 31 July-5 August 2011, pp. 119-124. doi:10.1109/ IJCNN.2011.6033209 (2011)

18. Ippolito, M.G., Telaretti, E., Zizzo, G., Graditi, G.: A new device for the control and the connection to the grid of combined RES-based generators and electric storage systems. In: Proceedings of IEEE International Conference on Clean Electrical Power (ICCEP'13), Alghero, Italy, 11-13 June, 2013, pp. 262-267. doi:10.1109/ ICCEP.2013.6586999 (2013)

19. Italian Ministry for Economic Development Decree 79/99: Attuazione della direttiva 96/92/CE recante norme comuni per il mercato interno dell'energia elettrica, 31 March 1999

20. Johnsen, T.A., Olsen, O.J.: The Relationship Between Wholesale and Retail Electricity Prices to Households in the Nordic Countries. University of Vaasa, VaasaEMG (2008)

21. Lee, T.Y.: Operating schedule of battery energy storage system in a time-of-use rate industrial user with wind turbine generators: a 
multipass iteration particle swarm optimization approach. IEEE Trans Energy Convers 22(3), 774-782 (2007). doi:10.1109/TEC. 2006.878239

22. Lijesen, M.G.: The real-time price elasticity of electricity. Energy Econ 29(2), 249-258 (2007). doi:10.1016/j.eneco.2006.08.008

23. Maly, D.K., Kwan, K.S.: Optimal battery energy storage system (BESS) charge scheduling with dynamic programming. IEE Proc Sci Meas Technol 142(6), 454-458 (1995). doi:10.1049/ip-smt: 19951929

24. Nguyen, M.Y., Nguyen, D.H., Yoon, Y.T.: A new battery energy storage charging/discharging scheme for wind power producers in real-time markets. Energies 5, 5439-5452 (2012). doi:10.3390/ en5125439

25. Public Utilities Commission of the State of California: Energy Division. Resolution E-4586. Permanent Load Shifting Program. 9 May 2013

26. Rupanagunta, P., Baughman, M.L., Jones, J.W.: Scheduling of cool storage using non-linear programming techniques. IEEE Trans Power Syst 10(3), 1279-1285 (1995). doi:10.1109/59.466526

27. Schoenung, S.M., Hassenzahl, W.V.: Long-vs. short term energy storage technologies analysis. Technical Report. SAND2003-2783. Sandia National Laboratories, Albuquerque, August 2003

28. Sioshansi, R., Denholm, P., Jenkin, T., Weiss, J.: Estimating the value of electricity storage in PJM: arbitrage and some welfare effects. Energy Econ 31(2), 269-277 (2009). doi:10.1016/j.eneco. 2008.10.005

29. Telaretti, E., Dusonchet, L., Massaro, F., Mineo, L., Pecoraro, G., Milazzo, F.: A simple operation strategy of battery storage systems under dynamic electricity pricing: an Italian case study for a medium-scale public facility. In: Proceedings of the 3rd Renewable Power Generation Conference (RPG'14), 24-25 September 2015, Naples, Italy, pp. 1-7. doi:10.1049/cp.2014.0842 (2014)
30. Van de Ven, P., Hegde, N., Massoulié, L., Salonidis, T.: Optimal control of residential energy storage under price fluctuations. In: Proceedings of the International Conference on Smart Grids, Green Communications and IT Energy-aware Technologies, Venice, Italy, May 2011, pp. 159-162 (2011)

31. Viswanathan, V., Kintner-Meyer, M., Balducci, P., Jin, C.: National Assessment of Energy Storage for grid balancing and arbitragevol. 2: cost and performance characterization, U.S., Technical Report. Department of Energy, Pacific Northwest National Laboratory, Richland, WA, September 2013

32. Yoo, J., Park, B., An, K., Al-Ammar, E.A., Khan, Y., Hur, K., Kim, J.H.: Look-ahead energy management of a grid-connected residential PV system with energy storage under time-based rate programs. Energies 5, 1116-1134 (2012). doi:10.3390/en5041116

33. Youn, L.T., Cho, S.: Optimal operation of energy storage using linear programming technique. In: Proceedings of the World Congress on Engineering and Computer Science (WCECS'09), 20-22 October 2009, San Francisco, pp. 480-485 (2009)

34. Zheng, M., Meinrenken, C.J., Lackner, K.S.: Agent-based model for electricity consumption and storage to evaluate economic viability of tariff arbitrage for residential sector demand response. Appl Energy 126, 297-306 (2014). doi:10.1016/j.apenergy.2014. 04.022 